\title{
Cytosolic THUMPD1 promotes breast cancer cells invasion and metastasis via the AKT-GSK3-Snail pathway
}

\author{
Xiupeng Zhang ${ }^{1}$, Guiyang Jiang ${ }^{1}$, Mingfang Sun ${ }^{1}$, Haijing Zhou ${ }^{1}$, Yuan Miao ${ }^{1}$, \\ Mengyuan Liang ${ }^{1}$, Enhua Wang ${ }^{1}$, Yong Zhang ${ }^{2}$ \\ ${ }^{1}$ Department of Pathology, First Affiliated Hospital and College of Basic Medical Sciences, China Medical University, Shenyang, \\ China \\ ${ }^{2}$ Department of Pathology, Cancer Hospital of China Medical University, Shenyang, China
}

Correspondence to: Yong Zhang, email: zhangyong@cancerhosp-In-cmu.com

Keywords: THUMPD1, breast cancer, AKT signaling, snail, GSK3 $\beta$

Received: October 05, 2016

Accepted: December 27, 2016

Published: January 05, 2017

\section{ABSTRACT}

\begin{abstract}
Human THUMP domain-containing protein 1 (THUMPD1) is a specific adaptor protein that modulates tRNA acetylation through interaction with NAT10. Immunohistochemical analysis of 146 breast cancer specimens ( 82 triple-negative and 64 non-triple-negative cases) indicated THUMPD1 expression is higher in breast cancer tissues $(60.9 \%, 89 / 146)$ than normal breast tissues $(28.3 \%, 15 / 53$; $p<0.001)$. Overall and cytosolic, but not nuclear, THUMPD1 expression in breast cancer correlated with advanced TNM stage $(p=0.003$ and $p<0.001$, respectively), lymph node metastasis $(p=0.001$ and $p<0.001$, respectively), and poor patient prognosis ( $p=0.001$ and $p<0.001$, respectively). THUMPD1 interacted and colocalized with YAP, but did not affect Hippo pathway activity. THUMPD1 overexpression enhanced breast cancer cells invasion and migration in vivo and in vitro, possibly through activation of AKT, GSK3 $\beta$ and Snail, and inhibition of E-cadherin. Treatment with the AKT inhibitor, LY294002, reduced the effects of THUMPD1 overexpression in breast cancer cells. These results indicate that THUMPD1 promotes breast cancer cells invasion and migration via the AKT-GSK3 $\beta$-Snail pathway.
\end{abstract}

\section{INTRODUCTION}

Breast cancer is the leading cause of cancer death in women $[1,2]$. Despite advances in tumor diagnostic and therapeutic strategies, patient outcomes are still poor due in part to cancer cell metastasis and heterogeneity [3, 4]. Novel biomarkers are needed to more accurately identify high-risk patients and to predict disease prognosis [5]. Genes abnormally expressed during tumor progression and metastasis may be used as markers to provide prognostic information beyond standard clinical assessment $[6,7]$.

Human THUMP domain-containing protein 1 (THUMPD1) is a specific adaptor protein that interacts with NAT10, a human acetyltransferase involved in histone and microtubule modification, and thus modulates tRNA acetylation [8]. Havugimana, et al. [9] suggested that THUMPD1 interacted with Yes-associated protein (YAP), a major transcriptional co-activator of the Hippo pathway, which plays crucial roles in tumor proliferation and invasion [10-13]. Our preliminary experiments showed that THUMPD1 expression was elevated in breast carcinoma samples as compared to normal breast tissues. However, THUMPD1 downstream signaling pathways in human breast cancer are as yet largely unknown. We hypothesized that THUMPD1 may promote malignant transformation through its interaction with YAP.

To test this hypothesis, we examined THUMPD1 expression and localization in breast cancer tissues and investigated associations between THUMPD1 subcellular localization and patient clinicopathological factors. We also addressed the effects of THUMPD1 on cancer cell migration and invasion, and analyzed potential downstream signaling pathways.

\section{RESULTS}

THUMPD1 expression and subcellular localization in breast carcinoma

We performed immunohistochemistry to assess THUMPD1 expression in 146 breast cancer and 53 paired noncancerous specimens. THUMPD1 presented 
negative or weak nuclear expression in normal breast tissues (Figure 1A-1B), moderate nuclear expression in carcinoma in situ samples (Figure 1C), and strong nuclear expression in IDCs (Figure 1D), which also showed positive THUMPD1 cytosolic expression (Figure 1E). Positive THUMPD1 expression frequency was higher in IDCs $(60.9 \%, 89 / 146)$ than in normal breast ductal epithelium $(28.3 \%, 15 / 53, p<0.001$; Figure 1F). Cytosolic and nuclear THUMPD1 expression frequency were $37 \%$ and $37.7 \%$, respectively, in breast cancers, and $24.5 \%$ $(p=0.093)$ and $5.6 \%(p<0.001)$, respectively, in normal breast tissues. Statistical analyses revealed that overall and cytosolic THUMPD1 expression correlated with high TNM stage ( $p=0.003$ and $p<0.001$, respectively) and lymph node metastasis $(p=0.001$ and $p<0.001$, respectively). However, nuclear THUMPD1 expression showed no obvious correlation with clinicopathological factors (Table 1). Kaplan-Meier analysis indicated reduced patient survival in tumors positive for overall and cytosolic THUMPD1 expression (131.86 \pm 4.71 months and $121.35 \pm 7.33$ months, respectively) as compared with THUMPD1-negative patients $(148.11 \pm 1.88$ months, $p=0.001$, and $148.05 \pm 1.37$ months, $p<0.001$, respectively; Figure $1 \mathrm{G}-1 \mathrm{H})$. Survival of patients with and without nuclear THUMPD1 was similar $(136.50 \pm$ 5.32 months versus $139.26 \pm 3.64$ months, respectively, $p$ $=0.653$; Figure 1I). However, subsequent cox univariate (UA) and multivariate (MA) analyses revealed that cytosolic overexpression of THUMPD1 could not be considered an independent prognostic factor in breast cancer ( $p=0.001$ for UA, and $p=0.146$ for MA, Table 2).

We then assessed THUMPD1 expression and subcellular localization in cell lines through western blotting and immunofluorescence. All breast cancer cell lines were positive for THUMPD1 expression, whereas the normal breast ductal cell line, MCF-10A, showed only weak expression (Figure 2A). Breast cancer cells exhibited both cytosolic and nuclear THUMPD1 expression, with only nuclear expression in MCF-10A cells (Figure 2B). In MCF-7 cells transfected with the THUMPD1-myc expression plasmid, exogenous THUMPD1 was largely localized in the cytoplasm (Figure 2C).
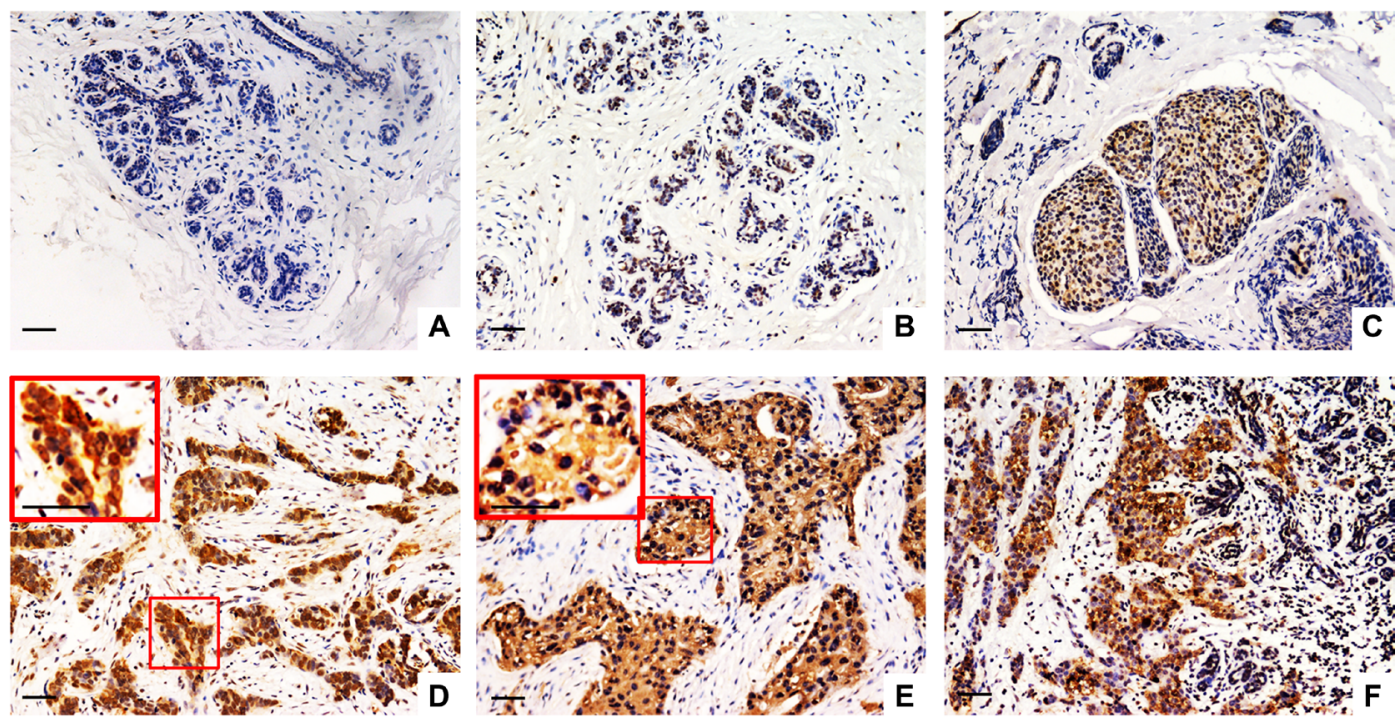

$\mathbf{G}$

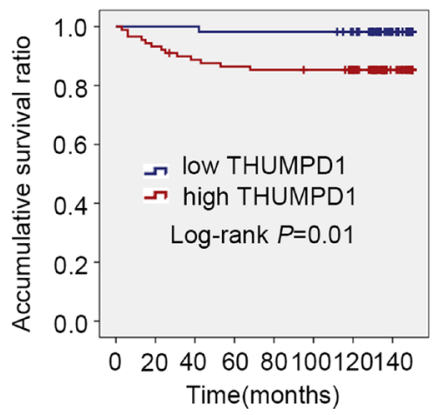

H

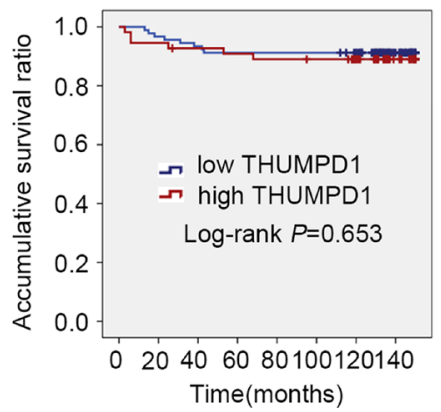

I

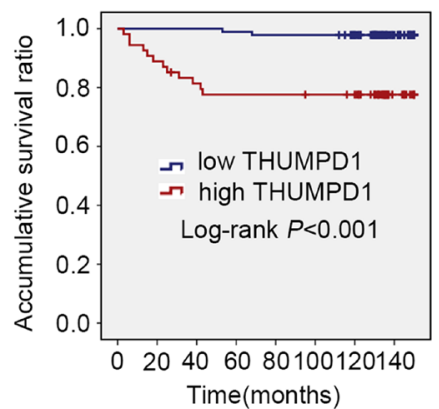

Figure 1: THUMPD1 expression and subcellular localization in breast tumors, and association with patient survival. THUMPD1 subcellular localization as shown by immunohistochemistry. In normal breast ductal cells, THUMPD1 expression was either absent (A) or weak (B). In carcinoma in situ (C) and IDC cells (D), THUMPD1 was observed in the nucleus and cytoplasm at moderate and high levels, respectively. In some IDC specimens, THUMPD1 was exclusively localized in the cytoplasm (E). THUMPD1 cytosolic and nuclear expression was higher in IDC (F) than in normal breast ductal cells. Magnification, $\times 200$ and $\times 400$. Kaplan-Meier analysis demonstrated that patient overall survival negatively correlated with overall $(\mathbf{G})$ and cytosolic $(\mathbf{H})$, but not nuclear (I), THUMPD1 expression. 
Table 1: Correlation of THUMPD1 overexpression with clinicopathological features in 146 cases breast cancer

\begin{tabular}{|c|c|c|c|c|c|c|c|c|c|c|c|c|}
\hline \multirow{2}{*}{ Clinicopathological factors } & \multicolumn{4}{|c|}{ The overall expression } & \multicolumn{4}{|c|}{ Cytosolic expression } & \multicolumn{4}{|c|}{ Nuclear expression } \\
\hline & $N$ & positive & negative & $p$ & $N$ & positive & negative & $p$ & $N$ & positive & negative & $p$ \\
\hline \multicolumn{13}{|l|}{ Age } \\
\hline$<52$ & 75 & 48 & 27 & \multirow{2}{*}{0.499} & 75 & 29 & 46 & \multirow{2}{*}{0.733} & 75 & 27 & 48 & \multirow{2}{*}{0.734} \\
\hline$\geq 52$ & 71 & 41 & 30 & & 71 & 25 & 46 & & 71 & 28 & 43 & \\
\hline \multicolumn{13}{|l|}{ TNM classification } \\
\hline $\mathrm{I}+\mathrm{II}$ & 122 & 68 & 54 & \multirow{2}{*}{0.003} & 122 & 36 & 86 & \multirow{2}{*}{$<0.001$} & 122 & 44 & 78 & \multirow{2}{*}{0.368} \\
\hline III & 24 & 21 & 3 & & 24 & 18 & 6 & & 24 & 11 & 13 & \\
\hline \multicolumn{13}{|l|}{ Lymph node metastasis } \\
\hline positive & 30 & 26 & 4 & \multirow{2}{*}{0.001} & 30 & 20 & 10 & \multirow{2}{*}{$<0.001$} & 30 & 16 & 14 & \multirow{2}{*}{0.058} \\
\hline negative & 116 & 63 & 53 & & 116 & 34 & 82 & & 116 & 39 & 77 & \\
\hline \multicolumn{13}{|l|}{ Triple-negative(ER,PR,Her-2) } \\
\hline positive & 82 & 54 & 28 & \multirow{2}{*}{0.177} & 82 & 20 & 44 & \multirow{2}{*}{0.23} & 82 & 31 & 51 & \multirow{2}{*}{1} \\
\hline negative & 64 & 35 & 29 & & 64 & 34 & 48 & & 64 & 24 & 40 & \\
\hline
\end{tabular}

A
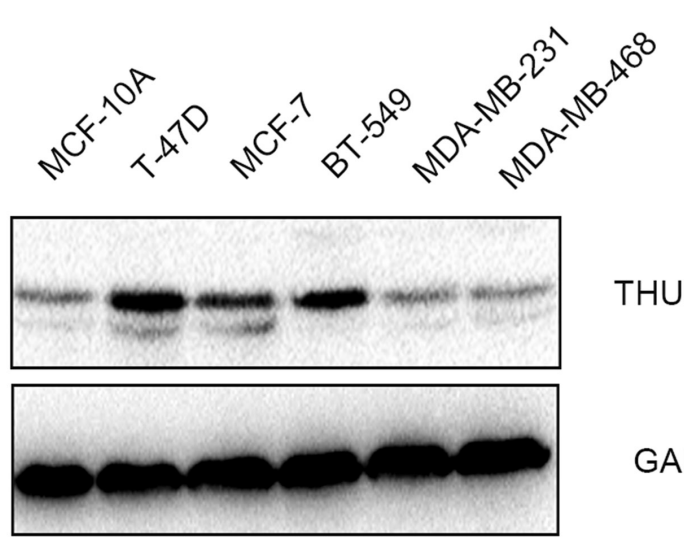

THUMPD1

GAPDH

B

MCF-10A MCF-7 BT-549 MDA-MB-468
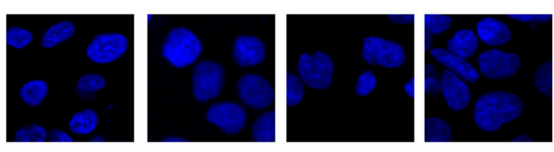

DAPI
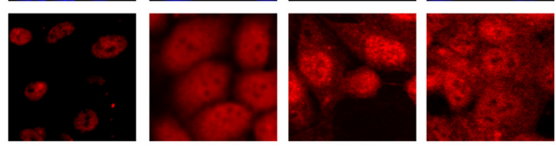

THUMPD1
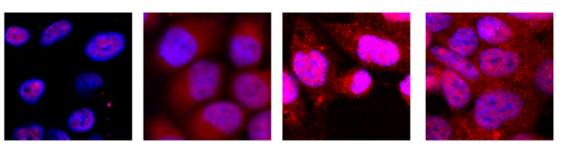
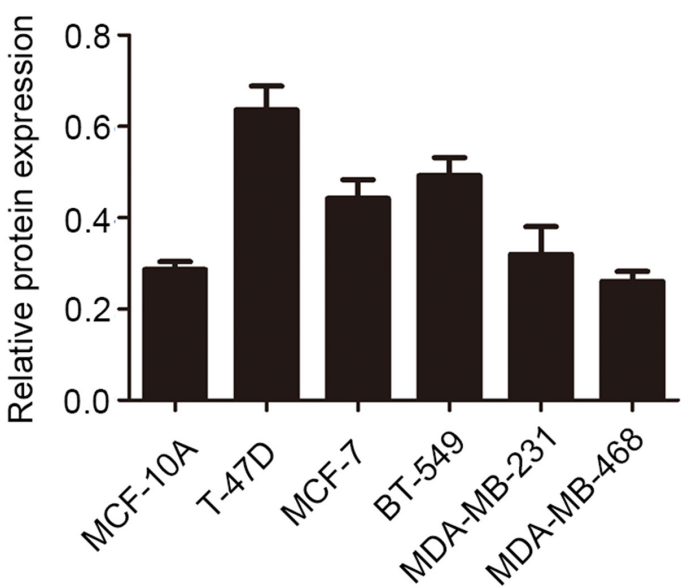

C

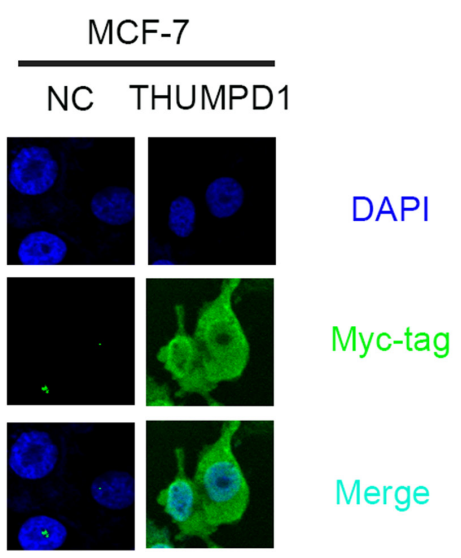

Figure 2: THUMPD1 expression and subcellular localization in breast cancer cell lines. THUMPD1 expression was higher in most tested breast cancer cell lines compared to normal breast cells (MCF-10A), but was lower in MDA-MB-231 and MDA-MB-468 cells (A). Immunofluorescence analyses indicated that THUMPD1 was mainly localized in MCF-10A cell nuclei, and in both nuclei and cytoplasm of breast cancer cells (B). Immunofluorescence analysis of THUMPD1-myc expression in MCF-7 cells using a Myc-tag antibody (C). Overexpressed THUMPD1 was predominantly localized in the cytoplasm. No positive signal was detected in untransfected cells. Magnification, $\times 600$. 
Table 2: Summary of Cox univariate and multivariate regression analysis of the association between clinicopathological features and overall survival in 146 cases of breast cancer

\begin{tabular}{lcc}
\hline \multicolumn{1}{c}{ Clinicopathological feature } & Hazard ratio $\mathbf{( 9 5 \%}$ CI) & $\boldsymbol{P}$ \\
\hline Univariate analysis & & 0.582 \\
Age & $0.582(0.195-1.728)$ & $<0.001$ \\
TNM classification & $96.688(12.594-742.282)$ & $<0.001$ \\
Lymph node metastasis & $66.491(8.679-509.418)$ & 0.967 \\
Triple-negative & $1.023(0.355-2.948)$ & 0.654 \\
Nuclear expression & $1.274(0.442-3.671)$ & 0.001 \\
Cytosolic expression & $11.771(2.632-52.635)$ & 0.034 \\
The overall expression & $8.984(1.175-68.686)$ & \\
Multivariate analysis & & 0.023 \\
TNM classification & $16.978(1.485-194.07)$ & 0.107 \\
Lymph node metastasis & $7.408(0.649-84.579)$ & 0.146 \\
Cytosolic expression & $4.531(0.59-34.793)$ & 0.889 \\
The overall expression & $0.821(0.052-13.033)$ & \\
\hline
\end{tabular}

\section{Interaction between THUMPD1 and YAP}

Immunoprecipitation and immunofluorescence were performed using THUMPD1-overexpressing MCF-7 cells to investigate the interaction between THUMPD1 and YAP. Exogenous THUMPD1 interacted directly with YAP (Figure 3A), and expression was co-localized in both the cytoplasm and nucleus (Figure 3B).

We assessed YAP phosphorylation status and distribution in THUMPD1-overexpressing MCF-7 and MDA-MB-468 cells, and in THUMPD1-deficient MCF-7 and BT-549 cells. Neither overexpression nor depletion of THUMPD1 affected levels of YAP, p-YAP, or the upstream regulator, LATS and p-LATS1 (Figure 3C). YAP and p-YAP subcellular distributions (Figure 3D) were also unchanged. Therefore, THUMPD1, despite its interaction with YAP, may not influence Hippo signaling.

\section{THUMPD1 enhanced breast cancer cells invasion and migration}

We then addressed THUMPD1 effects on tumor cell invasion and migration by overexpressing THUMPD1 in MCF-7 and MDA-MB-468 cells or depleting THUMPD1 in MCF-7 and BT-549 cells. In MCF-7 and MDA-MB-468 cells, THUMPD1 overexpression enhanced migration ( $p=0.014$ and $p=0.008$, respectively; Figure 4A) and invasion $(p=0.048$ and $p=0.014$, respectively; Figure 4B). In MCF-7 and BT-549 cells, THUMPD1 depletion inhibited migration $(p=0.020$ and $p=0.032$, respectively; Figure 4A) and invasion $(p=0.033$ and $p=0.004$, respectively, Figure 4B). In nude mice intravenously injected with THUMPD1-overexpressing MCF-7 cells, lung metastasis incidence was increased compared with controls ( $4 / 4$ versus $2 / 4$ in control), as were total numbers of lung metastatic nodules ( $p=0.015$; Figure 4C).

\section{THUMPD1 downregulated E-cadherin via AKT- GSK3ק-Snail signaling}

We analyzed expression of proteins involved in breast cancer cell epithelial-mesenchymal transition (EMT), with or without THUMPD1 overexpression or knockdown. Western blotting results revealed that THUMPD1 overexpression in MCF-7 and MDA-MB-468 cells upregulated Snail and downregulated E-cadherin (Figure 5A).THUMPD1 knockdown in MCF-7 and BT-549 cells downregulated Snail and upregulated E-cadherin. Levels of other proteins like Slug, P120-catenin, $\beta$-catenin, $\alpha$-catenin, Occludin, Zo1 , N-cadherin, and Vimentin showed no obvious changes (Supplementary Figure 1A). THUMPD1-overexpressing cells treated with the proteasome inhibitor, MG132, showed enhanced Snail upregulation and E-cadherin downregulation (Supplementary Figure 1B), indicating that THUMPD1 may stabilize Snail protein levels.

We next examined whether THUMPD1 activated the key signaling pathway involved in Snail stabilization. THUMPD1 overexpression induced, and THUMPD1 knockdown reduced, $\mathrm{p}$-AKT and its downstream target, p-GSK3 $\beta$ (Figure 5B). The other key signaling proteins such as Active- $\beta$-catenin, p-P65, P65, p-ERK, ERK, p-JNK, JNK, p-P38, P38, p-FAK, and FAK showed no obvious changes (Supplementary Figure 1C). At the 
same time, AKT inhibition by LY294002 downregulated p-GSK3 $\beta$ and Snail, and upregulated E-cadherin expression (Figure 5C), indicating that THUMPD1 functional activity depends on PI3K/AKT/GSK3 $\beta$ signaling. Accordingly, breast cancer cell invasiveness was reduced following AKT inhibition (Figure 5D).

\section{DISCUSSION}

The present study showed that THUMPD1 is weakly expressed in normal breast cell nuclei, and strongly expressed in IDC cell nuclei. THUMPD1 is also expressed in the cytoplasm of IDC cells, and cytosolic THUMPD1 expression positively correlated with high TNM stage, lymph node metastasis, and poor patient prognosis. In cultured breast cancer cells, endogenous THUMPD1 also localized to both the cytoplasm and nucleus. Interestingly, exogenous THUMPD1 was found in the cytoplasm rather than the nucleus (Figures 1I and 3D), although the reasons for this are unclear, and must be addressed in future studies. We also examined THUMPD1 expression in both triple-negative and non-triple-negative breast cancers, and found no correlation between THUMPD1 distribution or expression and breast cancer type.

A previous study suggested that THUMPD1 might interact with the transcriptional regulator, YAP, a potential oncogene and a member of the Hippo signaling pathway [9]. Consistent with this, our results showed that THUMPD1 interacted and co-localized with YAP in both the cytoplasm and nucleus. However, changes in THUMPD1 expression had no obvious effects on Hippo pathway activity or YAP subcellular distribution. Nucleocytoplasmic shuttling of YAP may be responsible for THUMPD1 translocation; however, this hypothesis needs further investigation [10].

Our in vivo and in vitro experiments indicated that THUMPD1 enhanced breast cancer cells invasion and migration. THUMPD1 overexpression upregulated Snail and downregulated E-cadherin, suggesting that THUMPD1 may facilitate cancer invasion through Snail, which is an extremely unstable protein [14-16]. MG132, a proteasome inhibitor, further enhanced Snail expression, suggesting that THUMPD1 may stabilize Snail to promote breast cancer cells invasion. Previous studies demonstrated that the Wnt, transforming growth factor $\beta$ (TGF $\beta$ ), mitogen-activated protein kinase (MAPK), and phosphatidylinositol 3-kinase (PI3K)/AKT signaling pathways regulate Snail expression [17-24]. Furthermore, glycogen synthase kinase-3 $\beta$ (GSK3 $\beta$ ) and nuclear factor (NF)- $\kappa \mathrm{B}$ are key proteins controlling cancer metastasis [25-30]. Our results showed that THUMPD1 overexpression upregulated p-AKT and its downstream factor, p-GSK $3 \beta$. Phosphorylation inactivates GSK3 $\beta$, and this inactivation may stabilize Snail $[31,32]$. Our results
A

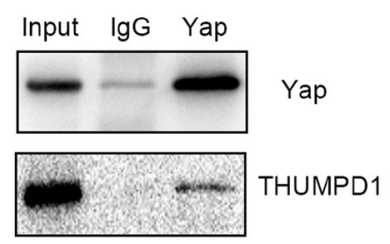

C

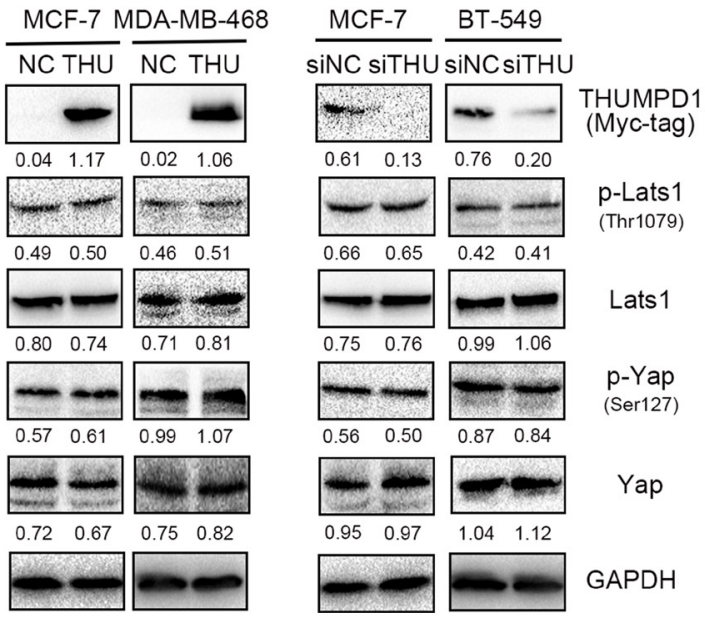

B

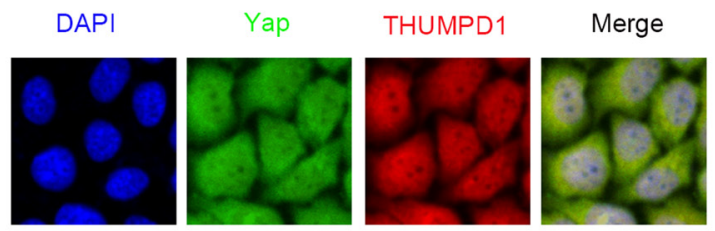

D

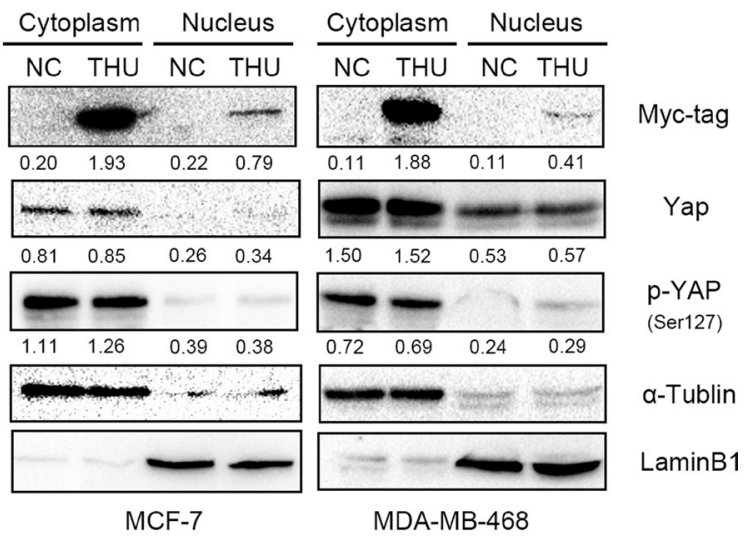

Figure 3: THUMPD1 interaction with YAP. Co-immunoprecipitation performed with a YAP antibody demonstrated that YAP bound THUMPD1 (A). Immunofluorescence analyses showed that THUMPD1 co-localized with YAP in the cytoplasm and nucleus (B). Magnification, $\times 600$. Western blotting analysis of THUMPD1-overexpressing MCF-7 and MDA-MD-468 cells, and THUMPD1deficient MCF-7 and BT-549 cells, revealed that YAP, p-YAP, LATS, and p-LATS levels were unaffected by THUMPD1 (C). THUMPD1 overexpression did not change YAP or p-YAP levels in MCF-7 and MDA-MD-468 cell cytoplasm or nuclei (D). 
revealed that AKT signaling inhibition by LY294002 downregulated Snail and upregulated E-cadherin, confirming that THUMPD1 decreased E-cadherin expression via the AKT-GSK3 $\beta$-Snail pathway.

Sharma, et al. demonstrated that THUMPD1 binds NAT10, which is a biomarker in several cancer types [8]. Ma, et al. indicated that NAT10 upregulation promotes hepatocellular carcinoma invasion by decreasing E-cadherin [34]. Zhang, et al. also confirmed that NAT10 enhanced colorectal cancer invasion and correlated with poor prognosis. We speculate that THUMPD1-
NAT10 binding could accelerate cancer cell invasion cooperatively. Alternatively, THUMPD1 may promote tumor invasion as a NAT10 downstream factor.

In conclusion, we observed THUMPD1 overexpression in the cytoplasm of breast cancer cells, which positively correlated with high TNM stage, lymph node metastasis, and poor patient prognosis. Although THUMPD1 interacted with YAP, no effects on other Hippo signaling pathway members were identified. THUMPD1 promoted breast cancer cells invasion and migration, and downregulated E-cadherin via the AKT-GSK3 $\beta$-Snail
A
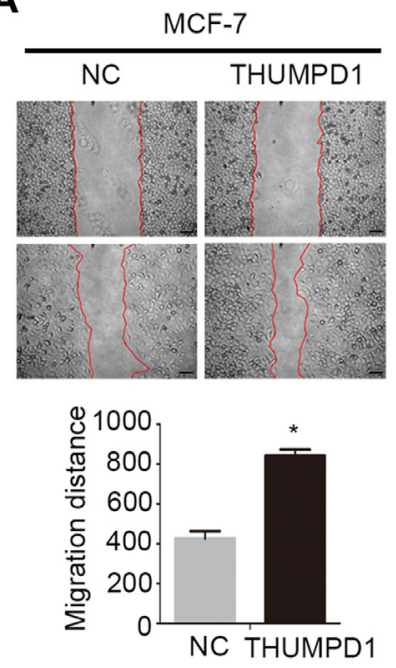

B
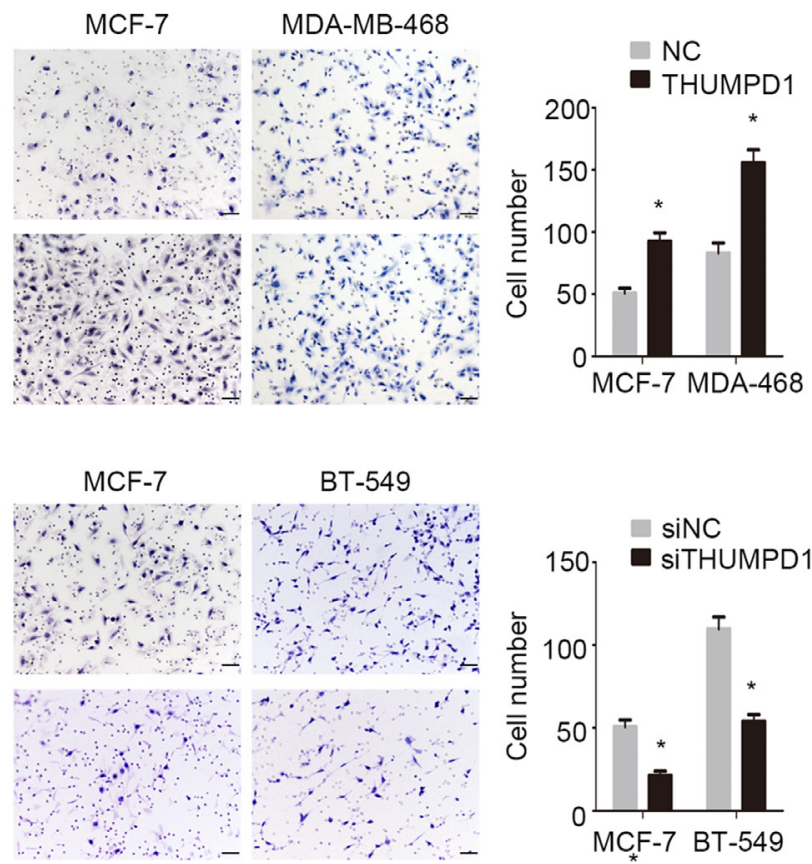
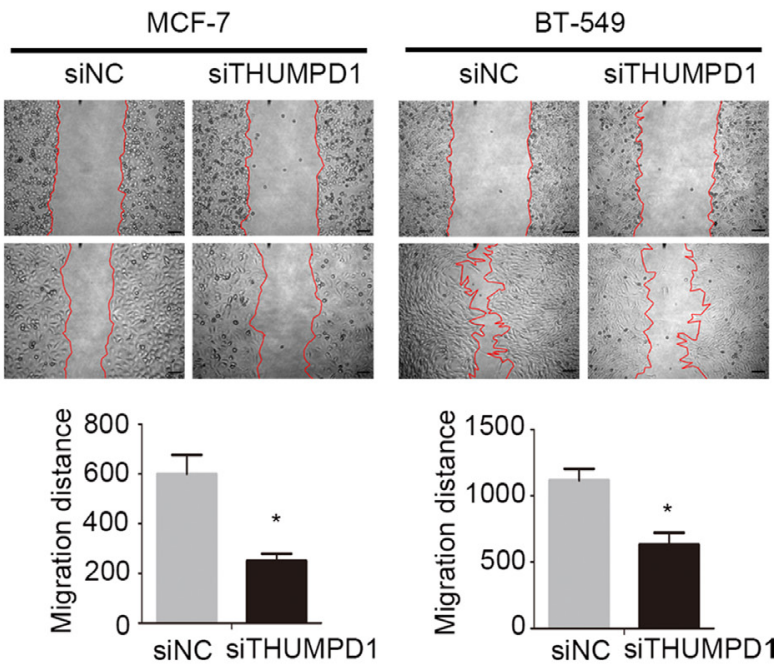

C

NC

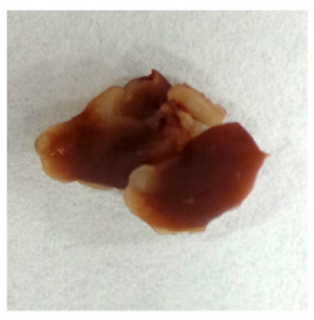

THUMPD1
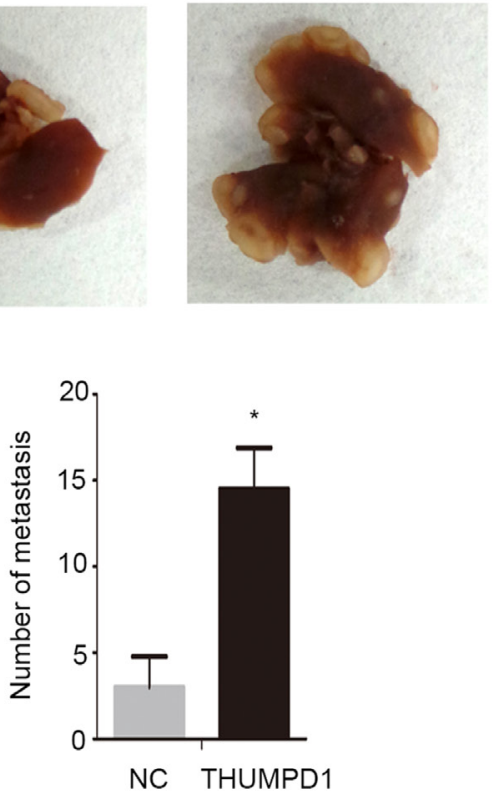

Figure 4: THUMPD1 enhanced breast cancer cell invasion and migration in vivo and in vitro. Cell migration (A) Cell invasion (B) THUMPD1 overexpression in MCF-7 and MDA-MB-468 cells enhanced migration and invasion, while THUMPD1 knockdown in MCF-7 and BT-549 cells inhibited migration and invasion. Mice injected with THUMPD1-overexpressing MCF-7 cells developed more pulmonary metastases than controls $(\mathbf{C}){ }^{*} p<0.05$. 
pathway, illuminating a possible mechanism underlying THUMPD1 in breast cancer progression.

\section{MATERIALS AND METHODS}

\section{Patients and clinical specimens}

The study protocol was approved by the institutional review board of China Medical University. All participants provided written informed consent, and the study was conducted according to the principles expressed in the Declaration of Helsinki. Primary tumor specimens were obtained from 146 breast cancer patients, including
82 triple-negative (deficient in estrogen receptor, progesterone receptor, and Her2/neu expression) and 64 non-triple-negative tumors. All patients diagnosed with invasive ductal carcinoma (IDC) underwent complete surgical resection at the Affiliated Cancer Hospital of China Medical University between 2001 and 2003. Among the 146 IDC specimens, there were 43 cases of ductal carcinoma in situ (DCIS). Complete follow-up data were available for all 146 analyzed cases. Patient survival was defined as the time from the day of surgery to the end of the follow-up period or the day of death due to recurrence or metastasis. None of the patients had received radiotherapy or chemotherapy before undergoing surgical
A

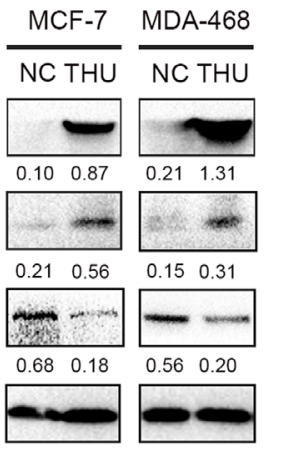

$\frac{\text { MCF-7 }}{\text { siNC siTHU }} \frac{\text { BT }-549}{\text { siNC siTHU }}$
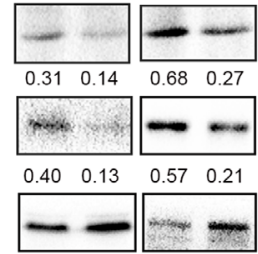

$\begin{array}{llll}0.46 & 0.71 & 0.18 & 0.43\end{array}$

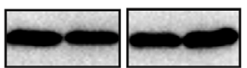

THUMPD1 (Myc-tag)

Snail

E-cadherin

GAPDH

C

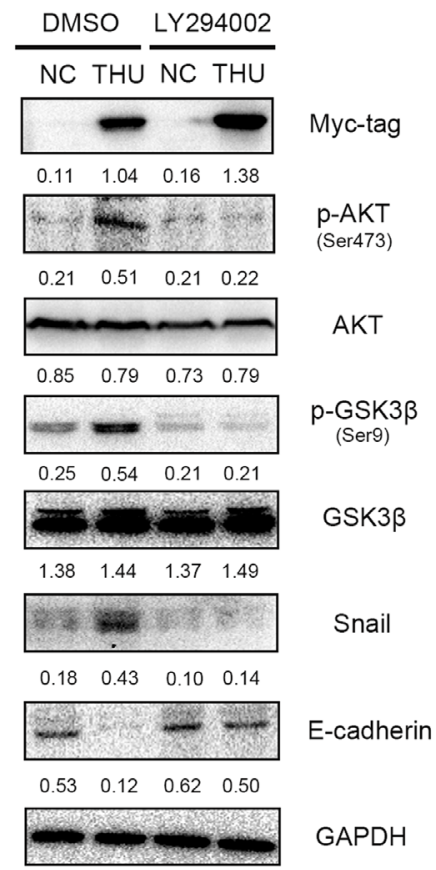

B

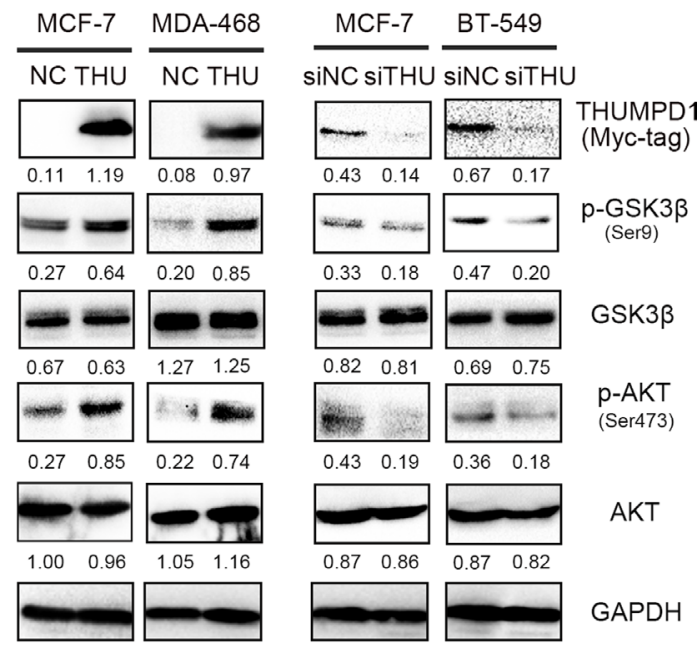

D
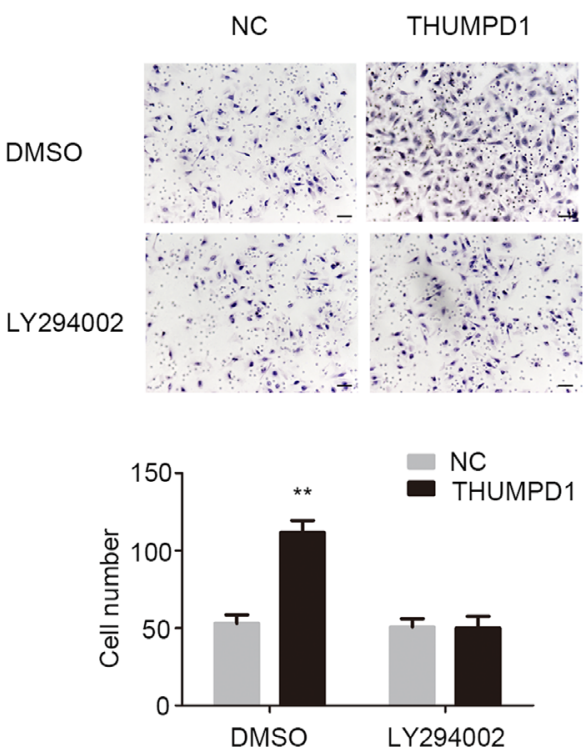

Figure 5: THUMPD1 promoted cancer cell invasion and migration via the AKT-GSK3ß-Snail pathway. THUMPD1 overexpression in MCF-7 and MDA-MB-468 cells upregulated Snail and downregulated E-cadherin, whereas THUMPD1 knockdown downregulated Snail and upregulated E-cadherin (A) p-AKT and its downstream target, p-GSK3 $\beta$, were increased in THUMPD1overexpressing MCF-7 and MDA-MD-468 cells, but decreased in THUMPD1-deficient MCF-7 and BT-549 cells (B) AKT inhibitor, LY294002 $(10 \mu \mathrm{M})$, reversed the effects of THUMPD1 on p-AKT and p-GSK3 $\beta$ expression, decreasing Snail and increasing E-cadherin (C) and reducing THUMPD1-overexpressing MCF-7 cell invasion (D). 
resection, and all patients were treated with routine chemotherapy after surgery.

\section{Cell lines}

MCF-10A, MCF-7, BT-549, MDA-MB-231, and MDA-MB-468 cell lines were obtained from Shanghai Cell Bank (Shanghai, China). All cells were cultured in RPMI 1640 medium (Invitrogen, Waltham, MA, USA) containing 10\% fetal calf serum (Invitrogen) and $100 \mathrm{IU} / \mathrm{mL}$ penicillin plus $100 \mu \mathrm{g} / \mathrm{mL}$ streptomycin (Sigma-Aldrich, St. Louis, MO, USA). Cells were grown in sterile culture dishes at $37^{\circ} \mathrm{C}$ in a $5 \% \mathrm{CO}_{2}$ atmosphere, and subcultured every two days using $0.25 \%$ trypsin (Invitrogen) for cell detachment.

\section{Immunohistochemistry}

All tissue specimens were fixed in neutral formaldehyde, embedded in paraffin, and sectioned (thickness, $4 \mu \mathrm{m}$ ). The streptavidin-peroxidase immunohistochemical method was used to improve staining. Tissue sections were incubated at $4^{\circ} \mathrm{C}$ overnight with THUMPD1 mouse monoclonal antibody (1:50 dilution; Santa Cruz Biotechnology, Inc., Dallas, TX, USA); phosphate-buffered saline was used as a blank control. Sections were then incubated with biotin-labeled secondary antibodies (Ultrasensitive; MaiXin, Fuzhou, China) at $37^{\circ} \mathrm{C}$ for $30 \mathrm{~min}$, followed by diaminobenzidine for coloration.

THUMPD1 staining intensity was scored as follows: 0 , none; 1 , weak (staining in $1-25 \%$ of cells); 2 , moderate $(26-50 \%) ; 3$, high $(51-75 \%$ of $)$, and 4 , very high (76-100\%). Individual tumor sample scores were multiplied to yield a final score of 0-12. THUMPD1 cytosolic expression was considered high in tumors with final scores $\geq 4$ and negative or weak in those with final scores $<4$. THUMPD1 nuclear localization was assessed in tumors with nuclear staining scores $>2$. In cases where THUMPD1 localized to either the cytoplasm or nucleus, tumors were considered THUMPD1-overexpressing.

\section{Western blotting and immunoprecipitation}

Western blotting and immunoprecipitation assays were performed as described previously [33]. Primary antibodies against THUMPD1 and LaminB1 (1:100) were purchased from Santa Cruz Biotechnology, Inc., and antibodies against GAPDH (1:500 and 1:5,000) were from Sigma (St. Louis, MO, USA). PI3 kinase inhibitor LY294002 $(10 \mu \mathrm{M})$ and antibodies against Snail, Slug, YAP, p-YAP-Ser127, LATS, p-LATS (Ser1079), Myctag, Vimentin, P38, p-P38, ERK, p-ERK, JNK, p-JNK, GSK3 $\beta$, p-GSK3 $\beta$ (Ser9), p-P65 (Ser536), AKT, p-AKT, and active $\beta$-catenin $(1: 1,000)$ were purchased from Cell Signaling Technology (Danvers, MA, USA). Antibodies against $\beta$-catenin, E-cadherin, $\mathrm{N}$-cadherin, P65, and P120 catenin $(1: 1,000)$ were purchased from BD Transduction Laboratories (Lexington, KY, USA). Antibodies against Zo-1 and Occludin (1:500) were purchased from Proteintech (Chicago, IL, USA). Proteasome inhibitor MG132 $(10 \mu \mathrm{M})$ and $\alpha$-tublin antibody (1:500) were purchased from Beyotime (Jiangsu, China).

\section{Plasmid construction and cell transfection}

The pCMV6-DDK-Myc empty vector and the pCMV6-DDK-Myc-THUMPD1 vector were purchased from OriGene (Rockville, MD, USA). THUMPD1-siRNA (sc-93083) and control siRNA (sc-37007) were purchased from Santa Cruz Biotechnology. Cells were transfected using the Lipofectamine 3000 kit (Invitrogen) according to the manufacturer's instructions.

\section{Immunofluorescence staining}

Cells were fixed with $4 \%$ paraformaldehyde, blocked with $1 \%$ bovine serum albumin, and incubated overnight with THUMPD1 monoclonal antibodies (1:100; Santa Cruz) at $4^{\circ} \mathrm{C}$. Cells were then incubated with tetramethylrhodamine isothiocyanate-conjugated secondary antibodies (Cell Signaling Technology) at $37^{\circ} \mathrm{C}$ for $2 \mathrm{~h}$. Cell nuclei were counterstained with $4^{\prime}$, 6-diamidino-2-phenylindole (DAPI). Epifluorescence microscopy was performed using an inverted Nikon TE300 microscope (Nikon Co., Ltd., Tokyo, Japan), and confocal microscopy was performed using a Radiance 2000 laser scanning confocal microscope (Carl Zeiss, Oberkochen, Germany).

\section{Matrigel invasion assay}

Cell invasion assays were performed using 24-well Transwell chambers with 8 - $\mu \mathrm{m}$ pores (Costar, Cambridge, MA, USA). Inserts were coated with $20 \mu$ l Matrigel (1:3 dilution; BD Bioscience, San Jose, CA, USA). Cells were trypsinized $48 \mathrm{~h}$ after transfection, resuspended at $3 \times 10^{5}$ cells in $100 \mu \mathrm{L}$ of serum-free medium, and transferred to the upper chambers of Transwell plates. Fetal bovine serum $(10 \%)$ was added to the lower chambers as chemoattractant. After $18 \mathrm{~h}$ incubation, cells that passed through the filter were fixed with $4 \%$ paraformaldehyde, stained with hematoxylin, and counted under a microscope in 10 randomly selected fields at $\times 40$ magnification.

\section{Wound healing assay}

Wounds were created in cell monolayers at $<90 \%$ confluence $48 \mathrm{~h}$ after transfection, using a $200-\mu \mathrm{l}$ pipette tip. Cell migration into the wound was observed at different time points. Wound areas were measured using Image $\mathrm{J}$ software, and representative images were taken. 
Each experimental condition was analyzed in duplicate, and three independent experiments were performed.

\section{Transplantation of tumor cells into nude mice}

Animals used in this study were treated according to the National Institutes of Health guide for the care and use of laboratory animals (NIH Publications No. 8023, revised 1978). Four-week-old female BALB/c nude mice were purchased from Slac (Shanghai, China) and kept in a laminar-flow cabinet under specific pathogenfree conditions for two weeks before use. Each mouse was then inoculated intravenously (tail vein) with $2 \times 10^{6}$ THUMPD1-transfected MCF-7 tumor cells in $0.2 \mathrm{~mL}$ sterile PBS. Six weeks after inoculation, mice were euthanized and examined for tumor growth and dissemination. Tumors, hearts, livers, lungs, and kidneys were dissected, fixed in 4\% formaldehyde (Sigma) and embedded in paraffin. Serial $6-\mu \mathrm{m}$-thick sections were cut, stained with hematoxylin and eosin, and examined by microscopy.

\section{Statistical analyses}

All statistical analyses were performed using SPSS 22.0 software (SPSS Statistics, Inc., Chicago, IL, USA). Immunohistochemistry results were analyzed via chisquare and Spearman's rank correlation tests. Kaplan-Meier survival analysis results were compared using the log-rank test. The Cox regression model was used to test prognostic values. All clinicopathological parameters were included in the Cox regression model and tested by univariate and multivariate analysis according to the enter method. Differences between groups were compared using Student $t$-tests, and $p<0.05$ was considered statistically significant.

\section{CONFLICTS OF INTEREST}

The authors declare no conflicts of interest.

\section{GRANT SUPPORT}

This work was supported by the National Natural Science Foundation of China (grant No.81602012 to X.Z., No. 81272606 and 81572854 to E.W., No. 81472805 to Y.M., and No. 81402369 to G. J.).

\section{REFERENCES}

1. Jemal A, Bray F, Center MM, Ferlay J, Ward E, Forman D. Global cancer statistics. CA Cancer J Clin. 2011; 2:69-90.

2. Liu Y, Ji R, Li J, Gu Q, Zhao X, Sun T, Wang J, Li J, Du Q, Sun B. Correlation effect of EGFR and CXCR4 and CCR7 chemokine receptors in predicting breast cancer metastasis and prognosis. J Exp Clin Cancer Res. 2010; 29:16.
3. Yan JD, Liu Y, Zhang ZY, Liu GY, Xu JH, Liu LY, Hu YM. Expression and prognostic significance of VEGFR-2 in breast cancer. Pathol Res Pract. 2015; 7:539-43.

4. Rakha EA. Pitfalls in outcome prediction of breast cancer. J Clin Pathol. 2013; 6:458-64.

5. Iqbal S, Vishnubhatla S, Raina V, Sharma S, Gogia A, Deo SS, Mathur S, Shukla NK. Circulating cell-free DNA and its integrity as a prognostic marker for breast cancer. Springerplus. 2015; 4:265.

6. van 't Veer LJ, Dai H, van de Vijver MJ, He YD, Hart AA, Mao M, Peterse HL, van der Kooy K, Marton MJ, Witteveen AT, Schreiber GJ, Kerkhoven RM, Roberts C, et al. Gene expression profiling predicts clinical outcome of breast cancer. Nature. 2002; 6871:530-6.

7. Parker JS, Mullins M, Cheang MC, Leung S, Voduc D, Vickery T, Davies S, Fauron C, He X, Hu Z, Quackenbush JF, Stijleman IJ, Palazzo J, et al. Supervised risk predictor of breast cancer based on intrinsic subtypes. J Clin Oncol. 2009; 8:1160-7.

8. Sharma S, Langhendries JL, Watzinger P, Kötter P, Entian KD, Lafontaine DL. Yeast Kre33 and human NAT10 are conserved $18 \mathrm{~S}$ rRNA cytosine acetyltransferases that modify tRNAs assisted by Tan1/THUMPD1. Nucleic Acids Res. 2015; 43:2242-58.

9. Havugimana PC, Hart GT, Nepusz T, Yang H, Turinsky AL, Li Z, Wang PI, Boutz DR, Fong V, Phanse S, Babu M, Craig SA, Hu P, et al. A census of human soluble protein complexes. Cell. 2012; 150:1068-81.

10. Wang Y, Dong Q, Zhang Q, Li Z, Wang E, Qiu X. Overexpression of yes-associated protein contributes to progression and poor prognosis of non-small-cell lung cancer. Cancer Sci. 2010; 101:1279-85.

11. Kim HM, Jung WH, Koo JS. Expression of Yes-associated protein (YAP) in metastatic breast cancer. Int J Clin Exp Pathol. 2015; 8:11248-57.

12. Wang Y, Xie C, Li Q, Xu K, Wang E. Clinical and prognostic significance of Yes-associated protein in colorectal cancer. Tumour Biol. 2013; 34:2169-74.

13. Xu MZ, Yao TJ, Lee NP, Ng IO, Chan YT, Zender L, Lowe SW, Poon RT, Luk JM. Yes-associated protein is an independent prognostic marker in hepatocellular carcinoma. Cancer. 2009; 115:4576-85.

14. Zhang X, Yu X, Jiang G, Miao Y, Wang L, Zhang Y, Liu Y, Fan C, Lin X, Dong Q, Han Q, Zhao H, Han Y, et al. Cytosolic TMEM88 promotes invasion and metastasis in lung cancer cells by binding DVLS. Cancer Res. 2015; 75:4527-37.

15. Bae GY, Hong SK, Park JR, Kwon OS, Kim KT, Koo J. Chronic TGF $\beta$ stimulation promotes the metastatic potential of lung cancer cells by Snail protein stabilization through integrin $\beta 3$-Akt-GSK3 $\beta$ signaling. Oncotarget. 2016; 7:25366-76. doi: 10.18632/oncotarget.8295.

16. Wang H, Wang HS, Zhou BH, Li CL, Zhang F, Wang XF, Zhang G, Bu XZ, Cai SH, Du J. Epithelial-mesenchymal 
transition (EMT) induced by TNF- $\alpha$ requires AKT/GSK$3 \beta$-mediated stabilization of snail in colorectal cancer. PLoS One. 2013; 8:e56664.

17. Zhang H, Sun JD, Yan LJ, Zhao XP. PDGF-D/PDGFR $\beta$ promotes tongue squamous carcinoma cell (TSCC) progression via activating p38/AKT/ERK/EMT signal pathway. Biochem Biophys Res Commun. 2016; 478:845-51.

18. Tashiro E, Henmi S, Odake H, Ino S, Imoto M. Involvement of the MEK/ERK pathway in EGF-induced E-cadherin down-regulation. Biochem Biophys Res Commun. 2016; 477:801-6.

19. Zhou B, Zhan H, Tin L, Liu S, Xu J, Dong Y, Li X, Wu L, Guo W. TUFT1 regulates metastasis of pancreatic cancer through HIF1-Snail pathway induced epithelialmesenchymal transition. Cancer Lett. 2016; 382:11-20.

20. Lv YF, Dai H, Yan GN, Meng G, Zhang X, Guo QN. Downregulation of tumor suppressing STF cDNA 3 promotes epithelial-mesenchymal transition and tumor metastasis of osteosarcoma by the Wnt/GSK-3 $\beta / \beta$-catenin/ Snail signaling pathway. Cancer Lett. 2016; 373:164-73.

21. Wu Y, Fu Y, Zheng L, Lin G, Ma J, Lou J, Zhu H, He Q, Yang B. Nutlin-3 inhibits epithelial-mesenchymal transition by interfering with canonical transforming growth factor$\beta 1$-Smad-Snail/Slug axis. Cancer Lett. 2014; 342:82-91.

22. Chen H, Zhu G, Li Y, Padia RN, Dong Z, Pan ZK, Liu K, Huang S. Extracellular signal-regulated kinase signaling pathway regulates breast cancer cell migration by maintaining slug expression. Cancer Res. 2009;69:9228-35.

23. Suh Y, Yoon CH, Kim RK, Lim EJ, Oh YS, Hwang SG, An S, Yoon G, Gye MC, Yi JM, Kim MJ, Lee SJ. Claudin-1 induces epithelial-mesenchymal transition through activation of the c-Abl-ERK signaling pathway in human liver cells. Oncogene. 2013; 32:4873-82.

24. Li Y, Che Q, Bian Y, Zhou Q, Jiang F, Tong H, Ke J, Wang K, Wan XP. Autocrine motility factor promotes epithelial-mesenchymal transition in endometrial cancer via MAPK signaling pathway. Int J Oncol. 2015; 47:1017-24.

25. Wu Y, Deng J, Rychahou PG, Qiu S, Evers BM, Zhou BP. Stabilization of snail by NF-kappaB is required for inflammation-induced cell migration and invasion. Cancer Cell. 2009; 15:416-28.

26. Yang CC, Wolf DA. Inflamed snail speeds metastasis. Cancer Cell. 2009; 15:355-7.
27. Lu Z, Ghosh S, Wang Z, Hunter T. Downregulation of caveolin-1 function by EGF leads to the loss of E-cadherin, increased transcriptional activity of beta-catenin, and enhanced tumor cell invasion. Cancer Cell. 2003 ; 4:499-515.

28. Saegusa M, Hashimura $M$, Kuwata $T$, Hamano $M$, Okayasu I. Crosstalk between NF-kappaB/p65 and betacatenin/TCF4/p300 signalling pathways through alterations in GSK-3beta expression during trans-differentiation of endometrial carcinoma cells. J Pathol. 2007; 213:35-45.

29. Song LB, Li J, Liao WT, Feng Y, Yu CP, Hu LJ, Kong QL, Xu LH, Zhang X, Liu WL, Li MZ, Zhang L, Kang TB, et al. The polycomb group protein Bmi-1 represses the tumor suppressor PTEN and induces epithelial-mesenchymal transition in human nasopharyngeal epithelial cells. J Clin Invest. 2009; 119:3626-36.

30. Lv W, Chen N, Lin Y, Ma H, Ruan Y, Li Z, Li X, Pan X, Tian X. Macrophage migration inhibitory factor promotes breast cancer metastasis via activation of HMGB1/TLR4/ NF kappa B axis. Cancer Lett. 2016; 375:245-55.

31. Zhang JP, Zeng C, Xu L, Gong J, Fang JH, Zhuang SM. MicroRNA-148a suppresses the epithelial-mesenchymal transition and metastasis of hepatoma cells by targeting Met/Snail signaling. Oncogene. 2014; 33:4069-76.

32. Zhou BP, Deng J, Xia W, Xu J, Li YM, Gunduz M, Hung MC. Dual regulation of Snail by GSK-3beta-mediated phosphorylation in control of epithelial-mesenchymal transition. Nat Cell Biol. 2004; 6:931-40.

33. Yu X, Zhang X, Zhang Y, Jiang G, Mao X, Jin F. Cytosolic TMEM88 promotes triple-negative breast cancer by interacting with Dvl. Oncotarget. 2015; 6:25034-45. doi: 10.18632/oncotarget.4379.

34. Ma R, Chen J, Jiang S, Lin S, Zhang X, Liang X. Up regulation of NAT10 promotes metastasis of hepatocellular carcinoma cells through epithelial-to-mesenchymal transition. Am J Transl Res. 2016; 10:4215-4223.

35. Zhang H, Hou W, Wang HL, Liu HJ, Jia XY, Zheng XZ, Zou YX, Li X, Hou L, McNutt MA, Zhang B. GSK-3 $\beta$ regulated $\mathrm{N}$-acetyltransferase 10 is involved in colorectal cancer invasion. Clin Cancer Res. 2014; 17:4717-29. 